\title{
Cost-Effectiveness Assessment of Orphan Drugs
}

\section{A Scientific and Political Conundrum}

\author{
Steven Simoens • Eline Picavet • Marc Dooms • \\ David Cassiman · Thomas Morel
}

Published online: 18 December 2012

(C) Springer International Publishing Switzerland 2012

In September 2012, the Dutch Healthcare Insurance Board issued advice not to reimburse orphan drugs that target lysosomal storage disorders: agalsidase alfa and agalsidase beta for Fabry disease and alglucosidase alfa for Pompe disease [1, 2].

With respect to the former, the Healthcare Insurance Board concluded that enzyme replacement therapy offered an added therapeutic value compared with symptomatic treatment of Fabry disease [1]. However, enzyme replacement therapy was not cost effective at an incremental cost of $€ 3.3$ million per quality-adjusted life-year gained. The unfavourable cost effectiveness originated from the limited additional therapeutic value (as a result of the slow progression of the disease) and high costs: enzyme replacement therapy costs $€ 200,000$ per patient per year, resulting in a total budget impact of $€ 11$ million per year for the Netherlands. The Healthcare Insurance Board argued that continued reimbursement of enzyme replacement therapy for Fabry disease would imply that limited resources are not available to reimburse other, more cost effective health technologies.

With respect to the latter, the Healthcare Insurance Board recommended to restrict reimbursement of

S. Simoens $(\varangle) \cdot$ E. Picavet · T. Morel

Department of Pharmaceutical and Pharmacological Sciences,

KU Leuven, Onderwijs en Navorsing 2, Herestraat 49,

P.O. Box 521, 3000 Leuven, Belgium

e-mail: steven.simoens@pharm.kuleuven.be

\section{Dooms}

Hospital Pharmacy, University Hospitals Leuven,

Leuven, Belgium

D. Cassiman

Department of Hepatology, University Hospitals Leuven,

Leuven, Belgium alglucosidase alfa for Pompe disease to patients who suffer from the classic form of the disease and to patients who show symptoms of the (non-)classic disease during their infancy [2]. Alglucosidase alfa was found to generate an added therapeutic value over best supportive care, was cost effective at an incremental cost of $€ 0.3-0.9$ million per quality-adjusted life-year gained in the classic form of Pompe disease, but was not cost effective at an incremental cost of $€ 15$ million per quality-adjusted life-year gained in the non-classic form of Pompe disease. The annual cost of treating a patient with alglucosidase alfa amounted to $€ 0.4-0.7$ million.

\section{A Scientific Conundrum}

These two examples highlight some of the scientific challenges involved in assessing the cost effectiveness of orphan drugs.

Uncertainty tends to surround the safety and effectiveness of orphan drugs at the time of market launch. This clinical uncertainty may result from difficulties involved in recruiting a sufficient number of patients across countries, the lack of randomized controlled trials, and the use of surrogate efficacy measures [3]. As a result, there is an urgent need for the development of methodological guidelines about the assessment of the (cost) effectiveness of orphan drugs for (ultra-)rare diseases. In England and Wales, the National Institute for Health and Clinical Excellence (NICE) will be responsible for assessing orphan drugs from April 2013 onwards and has committed itself to developing methods to guide such an assessment [4].

Dutch physicians who are involved in treating patients with these lysosomal storage disorders have argued that the Healthcare Insurance Board is not able to issue an informed 
advice due to the lack of long-term data on safety and effectiveness [5]. For instance, on the occasion of the public hearing of the Dutch Healthcare Insurance Board on 21 September 2012, Professor Hollak stressed that the main challenge with enzyme replacement therapy is that effectiveness is different across patient subgroups [6]. While a clear benefit was shown in some patients, in others, such as those with end-renal or cardiac disease, a lesser benefit was observed. No conclusions can nonetheless be drawn as the overall amount of data remains too small, resulting from the low number of Dutch patients. To address this issue, the idea has been proposed to launch a compulsory Europeanwide registry following marketing authorization of an orphan drug [5]. The establishment of such registries for rare diseases is supported by the European Medicines Agency and by many member states, although registries are not without methodological limitations [3].

The ethical principle underlying economic evaluation is that scarce resources are allocated to the most cost-effective health technologies with a view to maximizing population health. The reimbursement of health technologies (such as orphan drugs) that tend to be not cost effective would provide a limited health gain in patients suffering from a rare disease, but reduce overall population health [7]. In this way, economic evaluation prefers to maximize the health of the total population rather than the health of a specific patient group. This, however, conflicts with the principle that each patient has a right of access to care, which is embedded in the Charter of Fundamental Rights and in legislation of the European Union [8, 9]. To explicitly take account of this principle in economic evaluation, approaches such as variable cost-effectiveness thresholds (which would set a higher threshold for orphan drugs) or weighted cost-effectiveness ratios (where the health gain in a patient with a rare disease would receive a higher weight) have been proposed in theory [3], but these approaches are not (yet) applied in decision making in practice.

\section{A Political Conundrum}

The decision-making process governing the assessment and reimbursement of orphan drugs is challenging for a number of reasons.

The possibility that (some) orphan drugs do not qualify for full reimbursement is not generally accepted. This suggestion was, for instance, opposed by the Dutch Societies for Clinical Genetics and for Paediatrics [5]. This is also reflected in the fact that all orphan drugs available in France are fully reimbursed, even though the French Transparency Commission has concluded that not all orphan drugs have an added clinical benefit compared with a relevant alternative [10]. In the absence of any notion of value in the decision-making process, it is clear that there is no need for assessing orphan drugs. Instead, we would argue that future research needs to elicit what society values about orphan drugs and then explicitly incorporate these value judgements in the decision-making process. With respect to the former, a discrete choice experiment can be conducted to identify and elicit the relative importance of the attributes of orphan drugs that matter to society. With respect to the latter, multi-criteria decision analysis can serve as a decision-making framework to consider how well (orphan) drugs perform on each of these attributes, the results of which can subsequently inform the reimbursement decision in a transparent and objective way [11].

In response to the uncertainty surrounding the (cost) effectiveness of orphan drugs, decision-making instruments such as conditional reimbursement or coverage with evidence development have been proposed [7]. These instruments grant initial reimbursement to orphan drugs subject to the commitment of collecting additional data, on the basis of which final reimbursement will be decided. Although this approach is theoretically appealing, in practice it is very difficult for a decision maker to stop reimbursement of an orphan drug that is used in clinical practice, if it later turns out to be not (cost) effective. It is noteworthy that a Dutch research project is currently being conducted into the risks of conditional reimbursement, with a specific focus on decisions to withdraw reimbursement [12].

Finally, the Dutch example shows how the decisionmaking process should not be organized: at the end of July, the draft advice by the Healthcare Insurance Board was leaked prior to its official release, leading to vocal opposition by members of parliament, physician associations, media and even health insurers themselves, and putting pressure on the health minister to not to follow the advice of the Healthcare Insurance Board. Instead of ad hoc reactions to a specific dossier, a societal debate needs to be launched about the value of orphan drugs themselves and compared with health technologies for non-rare diseases. To what extent does society wish to grant priority to treatments for rare diseases?

\section{Conclusions}

The reimbursement of orphan drugs for rare diseases and the role that the assessment of cost effectiveness plays in this decision is likely to give rise to continued debate among the different stakeholders (i.e., third-party payers, physicians, patients, health economists, ethicists, policy makers) who have an interest in the decision. In order to 
advance this debate, we believe that society needs to be better informed about the trade-offs involved in the decision whether to reimburse (orphan) drugs with a view to elicit what society values about (orphan) drugs and to explore how this can be explicitly taken into account in the decision-making process.

Acknowledgments No sources of funding were used to assist in the preparation of this manuscript. The authors have no conflicts of interest that are directly relevant to the content of this manuscript. DC received research funding and reimbursement of expenses from Sanofi-Genzyme, Shire and Actelion.

\section{References}

1. Advice on agalsidase alfa $\left(\right.$ Replagal $^{\circledR}$ ) and agalsidase beta $\left(\right.$ Fabrazyme $\left.^{\circledR}\right)$ for the indication 'Fabry disease'. Diemen: CVZ, 2012. http://www.cvz.nl/binaries/content/documents/cvzinternet/nl/ documenten/rubriek+zorgpakket/acp/1209/acp1209-7a-agalsidase. pdf. Accessed 17 Oct 2012.

2. Advice on alglucosidase alfa (Myozyme ${ }^{\circledR}$ ) for the indication 'Pompe disease'. Diemen: CVZ, 2012. http://www.cvz.nl/binaries/ content/documents/cvzinternet/nl/documenten/rubriek+zorgpakket/ acp/1209/acp1209-08a-alglucosidase.pdf. Accessed 17 Oct 2012.

3. Simoens S. Pricing and reimbursement of orphan drugs: the need for more transparency. Orphanet J Rare Dis. 2011;6:42.

4. NICE to assess high cost low volume drugs. London: Department of Health, 2012. http://www.dh.gov.uk/health/2012/07/very-highcost-drugs/. Accessed 17 Oct 2012.
5. Sheldon T. Dutch doctors call for EU evaluation of cost effectiveness of high cost orphan drugs. BMJ. 2012;345:e5461.

6. Argument of the Academic Medical Center in response to the preliminary advice from the Dutch Healthcare Insurance Board (CVZ) to stop reimbursement of Fabrazyme and Replagal. 2012. http://www.rarediseaseblogs.net/2012/09/24/argument-of-theacademic-medical-center-in-response-to-the-preliminary-advicefrom-the-dutch-healthcare-insurance-board-cvz-to-stop-reimbur sement-of-fabrazyme-and-replagal/. Accessed 25 Oct 2012.

7. Simoens S, Cassiman D, Dooms M, et al. Orphan drugs for rare diseases: is it time to revisit their special market access status? Drugs. 2012;72(11):1437-43.

8. Gericke CA, Riesberg A, Busse R. Ethical issues in funding orphan drug research and development. J Med Ethics. 2005;31(3): $164-8$.

9. European Commission. Regulation (EC) No 141/2000 of the European Parliament and of the Council of 16 December 1999 on orphan medicinal products. Off J Eur Commun. 2000; L18(1): $1-5$.

10. Simoens S, Dooms M. Market access of orphan drugs: one size fits all? Hosp Pharm Eur. 2012;62:59-63.

11. Hughes-Wilson W, Palma A, Schuurman A, et al. Paying for the orphan drug system: break or bend? Is it time for a new evaluation system for payers in Europe to take account of new rare disease treatments? Orphanet J Rare Dis. 2012;7(1):74.

12. ZonMw. The risks of conditional reimbursement: stopping can be more difficult than not starting! 2012. http://www.zonmw.nl/nl/ projecten/project-detail/the-risks-of-conditional-reimbursementstopping-can-be-more-difficult-than-not-starting/samenvatting/. Accessed 17 Oct 2012. 